NeJla Özmen (Düzce)

\title{
SOME NEW PROPERTIES OF GENERALIZED BESSEL POLYNOMIALS
}

Abstract. We obtain some new properties of generalized Bessel polynomials. We mainly investigate bilinear and bilateral generating function relations for these polynomials. We also derive a result giving certain families of bilateral generating functions for generalized Bessel polynomials and generalized Lauricella functions. We also discuss some special cases.

1. Introduction. In $[\mathrm{H}]$, Krall and Frink initiated a systematic investigation of Bessel polynomials and developed a theory of generalized Bessel polynomials

$$
\begin{aligned}
y_{n}(x, \alpha, \beta): & =\sum_{k=0}^{n}\left(\begin{array}{l}
n \\
k
\end{array}\right)\left(\begin{array}{c}
\alpha+n+k-2 \\
k
\end{array}\right) k !\left(\frac{x}{\beta}\right)^{k} \\
& ={ }_{2} F_{0}(-n, \alpha+n-1 ;-;-x / \beta) .
\end{aligned}
$$

Taking $\alpha=\beta=2$ in 1.1 , we get an explicit formula for the simple Bessel polynomials $y_{n}(x)$ given by

$$
\begin{aligned}
y_{n}(x) & :=\sum_{k=0}^{n}\left(\begin{array}{l}
n \\
k
\end{array}\right)\left(\begin{array}{c}
n+k \\
k
\end{array}\right) k !\left(\frac{x}{2}\right)^{k}=y_{n}(x, 2,2) \\
& ={ }_{2} F_{0}(-n, n+1 ;-;-x / 2) .
\end{aligned}
$$

These polynomials have the following generating function relations (see $[\mathrm{SM}])$ :

$$
\sum_{n=0}^{\infty} y_{n}(x, \alpha-n, \beta) \frac{t^{n}}{n !}=\left(1-\frac{x t}{\beta}\right)^{1-\alpha} \exp (t),
$$

2010 Mathematics Subject Classification: 33C45, 33C65.

Key words and phrases: generalized Bessel polynomials, generating function, bilinear and bilateral generating functions, recurrence relations, hypergeometric function.

Received 8 August 2018; revised 6 March 2019.

Published online 13 May 2019. 
and

$$
\begin{aligned}
\sum_{n=0}^{\infty} y_{n+m}(x & , \alpha-m-n, \beta) \frac{t^{n}}{n !} \\
& =\left(1-\frac{x t}{\beta}\right)^{1-\alpha} \exp (t) y_{m}\left(x\left(1-\frac{x t}{\beta}\right)^{-1}, \alpha-m, \beta\right) .
\end{aligned}
$$

In the literature, there are a lot of works relating to generalized Bessel polynomials and their applications (see, for instance, $\mathrm{RAB}, \mathrm{BSF}, \mathrm{CV}, \mathrm{BE}$, $\mathrm{CS}, \mathrm{DSS}, \mathrm{H}]$ ).

On the other hand, Lauricella functions are generalizations of Gauss hypergeometric functions to several variables. If $n$ is the number of variables, then four such generalizations, denoted by $F_{A}^{(n)}, F_{B}^{(n)}, F_{C}^{(n)}$, and $F_{D}^{(n)}$, were investigated by Lauricella $[\mathrm{L}]$. For $n=2$, these functions reduce to the Appell hypergeometric functions $F_{2}, F_{3}, F_{4}$ and $F_{1}$, respectively. Recall that the latter are defined by

$$
\begin{aligned}
F_{1}\left[a, b, b^{\prime} ; c ; x, y\right] & =\sum_{m, n=0}^{\infty} \frac{(a)_{m+n}(b)_{m}\left(b^{\prime}\right)_{n}}{(c)_{m+n}} \frac{x^{m}}{m !} \frac{y^{n}}{n !}, \\
F_{2}\left[a, b, b^{\prime} ; c, c^{\prime} ; x, y\right] & =\sum_{m, n=0}^{\infty} \frac{(a)_{m+n}(b)_{m}\left(b^{\prime}\right)_{n}}{(c)_{m}\left(c^{\prime}\right)_{n}} \frac{x^{m}}{m !} \frac{y^{n}}{n !}, \\
F_{3}\left[a, a^{\prime}, b, b^{\prime} ; c ; x, y\right] & =\sum_{m, n=0}^{\infty} \frac{(a)_{m}\left(a^{\prime}\right)_{n}(b)_{m}\left(b^{\prime}\right)_{n}}{(c)_{m+n}} \frac{x^{m}}{m !} \frac{y^{n}}{n !}, \\
F_{4}\left[a, b ; c, c^{\prime} ; x, y\right] & =\sum_{m, n=0}^{\infty} \frac{(a)_{m+n}(b)_{m+n}}{(c)_{m}\left(c^{\prime}\right)_{n}} \frac{x^{m}}{m !} \frac{y^{n}}{n !},
\end{aligned}
$$

where, as usual, $(\lambda)_{\nu}$ denotes the Pochhammer symbol given by (for $\lambda, \nu \in \mathbb{C}$ and in terms of the Gamma function)

$$
(\lambda)_{\nu}:=\frac{\Gamma(\lambda+\nu)}{\Gamma(\lambda)}= \begin{cases}1 & (\nu=0 ; \lambda \in \mathbb{C} \backslash\{0\}) \\ \lambda(\lambda+1) \ldots(\lambda+n-1) & (\nu=n \in \mathbb{N} ; \lambda \in \mathbb{C})\end{cases}
$$

with the convention $(0)_{0}:=1$.

A further generalization of the familiar Kampé de Fériet hypergeometric function in two variables, which is called the generalized Lauricella function (or the Srivastava and Daoust function), was introduced by Srivastava and Daoust $\mathrm{SD}$ :

$$
F_{C: D^{(1)} ; \ldots ; D^{(s)}}^{A: B^{(1)} ; \ldots ; B^{(s)}}\left(\begin{array}{l}
{\left[(a): \theta^{(1)}, \ldots, \theta^{(s)}\right]:\left[\left(b^{(1)}\right): \phi^{(1)}\right] ; \ldots ;\left[\left(b^{(s)}\right): \phi^{(s)}\right] ;} \\
{\left[(c): \psi^{(1)}, \ldots, \psi^{(s)}\right]:\left[\left(d^{(1)}\right): \delta^{(1)}\right] ; \ldots ;\left[\left(d^{(s)}\right): \delta^{(s)}\right] ;}
\end{array} z_{1}, \ldots, z_{s}\right)
$$




$$
=\sum_{m_{1}, \ldots, m_{s}=0}^{\infty} \Omega\left(m_{1}, \ldots, m_{s}\right) \frac{z_{1}^{m_{1}}}{m_{1} !} \ldots \frac{z_{s}^{m_{s}}}{m_{s} !}
$$

where

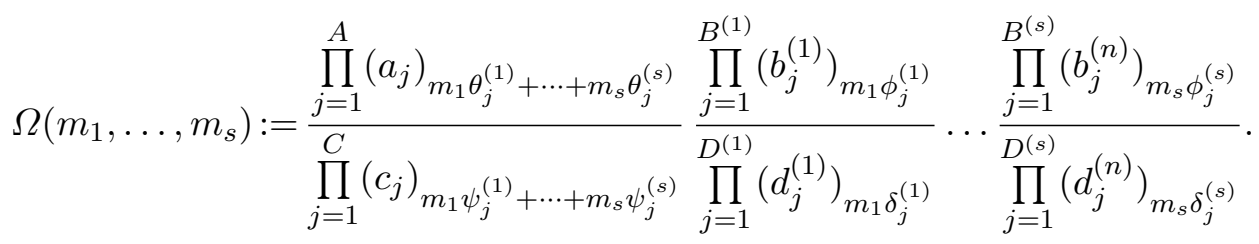

Here, the coefficients

$$
\begin{array}{ccc}
\theta_{j}^{(k)} & (j=1, \ldots, A ; k=1, \ldots, s), \quad \phi_{j}^{(k)} \quad\left(j=1, \ldots, B^{(k)} ; k=1, \ldots, s\right), \\
\psi_{j}^{(k)} & (j=1, \ldots, C ; k=1, \ldots, s), \quad \delta_{j}^{(k)} \quad\left(j=1, \ldots, D^{(k)} ; k=1, \ldots, s\right),
\end{array}
$$

are real constants and $\left(b_{B^{(k)}}^{(k)}\right)$ abbreviates the array of $B^{(k)}$ parameters

$$
b_{j}^{(k)} \quad\left(j=1, \ldots, B^{(k)} ; k=1, \ldots, s\right)
$$

with similar interpretations for other sets of parameters [SSHM]. For a bounded non-vanishing multiple sequence $\left\{\Omega\left(m_{1}, \ldots, m_{s}\right)\right\}_{m_{1}, \ldots, m_{s} \in \mathbb{N}_{0}}$ of real or complex parameters, define a function $\Psi_{n}\left(u_{1} ; u_{2}, \ldots, u_{s}\right)$ of $s$ (real or complex) variables $u_{1}, u_{2}, \ldots, u_{s}$ (see [SSHM] $)$ by

$$
\begin{aligned}
\Psi_{n}\left(u_{1} ; u_{2}, \ldots, u_{s}\right):= & \sum_{m_{1}=0}^{n} \sum_{m_{2}, \ldots, m_{s}=0}^{\infty} \frac{(-n)_{m_{1}}((b))_{m_{1} \phi}}{((d))_{m_{1} \delta}} \\
& \times \Omega\left(f\left(m_{1}, \ldots, m_{s}\right), m_{2}, \ldots, m_{s}\right) \frac{u_{1}^{m_{1}}}{m_{1} !} \ldots \frac{u_{s}^{m_{s}}}{m_{s} !}
\end{aligned}
$$

where, for convenience,

$$
((b))_{m_{1} \phi}=\prod_{j=1}^{B}\left(b_{j}\right)_{m_{1} \phi_{j}} \quad \text { and } \quad((d))_{m_{1} \delta}=\prod_{j=1}^{D}\left(d_{j}\right)_{m_{1} \delta_{j}} .
$$

In recent years, many researchers have studied multilinear and multilateral generating functions for different type of polynomials. For example, in [SL, Liu introduced bilateral generating functions for Lagrange polynomials and Lauricella functions. Similarly, in CCS the authors obtained bilateral generating functions for Chan-Chyan-Srivastava polynomials and generalized Lauricella functions. In 2012, they derived bilateral generating functions for Erkuş-Srivastava polynomials and generalized Lauricella functions (see [SSHM] $)$.Very recently, we have obtained bilateral generating func- 
tions for generalized Cesàro polynomials and generalized Lauricella functions (see $\mathrm{OE}]$ ). One can find various generating functions by the method of [N] and $\mathrm{NE}^{*}$.

The main object of this paper is to study several properties of generalized Bessel polynomials. Various families of multilinear and multilateral generating functions, their miscellaneous properties and also some special cases are given. In addition, we derive a result giving certain families of bilateral generating functions for generalized Bessel polynomials and generalized Lauricella functions.

\section{Bilinear, bilateral generating functions and special functions.} In this section, we derive several families of bilinear and bilateral generating functions for the generalized Bessel polynomials $y_{n}(x, \alpha, \beta)$ generated by using a similar method to that considered in $\mathrm{AE}$

We begin by stating the following theorem.

THEOREM 2.1. For non-identically-vanishing functions $\Omega_{\mu}\left(y_{1}, \ldots, y_{r}\right)$ of $r$ complex variables $y_{1}, \ldots, y_{r}(r \in \mathbb{N})$, indexed by complex numbers $\mu$, let

$$
\Lambda_{\mu, \psi}\left(y_{1}, \ldots, y_{r} ; \zeta\right):=\sum_{k=0}^{\infty} a_{k} \Omega_{\mu+\psi k}\left(y_{1}, \ldots, y_{r}\right) \zeta^{k} \quad\left(a_{k} \neq 0 ; \mu, \psi \in \mathbb{C}\right)
$$

and

$$
\begin{aligned}
\Theta_{n, p}^{\mu, \psi}\left(x, \alpha-n, \beta ; y_{1}, \ldots, y_{r} ; \xi\right) & \\
& :=\sum_{k=0}^{[n / p]} a_{k} y_{n-p k}(x, \alpha-n-p k, \beta) \Omega_{\mu+\psi k}\left(y_{1}, \ldots, y_{r}\right) \frac{\xi^{k}}{(n-p k) !} .
\end{aligned}
$$

Then, for $p \in \mathbb{N}$,

$$
\begin{aligned}
\sum_{n=0}^{\infty} \Theta_{n, p}^{\mu, \psi}(x, \alpha-n, \beta ; & \left.y_{1}, \ldots, y_{r} ; \frac{\eta}{t^{p}}\right) t^{n} \\
& =\left(1-\frac{x t}{\beta}\right)^{1-\alpha} \exp (t) \Lambda_{\mu, \psi}\left(y_{1}, \ldots, y_{r} ; \eta\right)
\end{aligned}
$$

provided that the series on both sides of (2.1) are convergent.

Proof. For convenience, let $S$ denote the left-hand side of (2.1). Then

$$
S=\sum_{n=0}^{\infty} \sum_{k=0}^{[n / p]} a_{k} y_{n-p k}(x, \alpha-n-p k, \beta) \Omega_{\mu+\psi k}\left(y_{1}, \ldots, y_{r}\right) \frac{\eta^{k}}{(n-p k) !} t^{n-p k} .
$$


Replacing $n$ by $n+p k$, we get

$$
\begin{aligned}
S & =\sum_{n=0}^{\infty} \sum_{k=0}^{\infty} a_{k} y_{n}(x, \alpha-n, \beta) \Omega_{\mu+\psi k}\left(y_{1}, \ldots, y_{r}\right) \eta^{k} \frac{t^{n}}{n !} \\
& =\sum_{n=0}^{\infty} y_{n}(x, \alpha-n, \beta) \frac{t^{n}}{n !} \sum_{k=0}^{\infty} a_{k} \Omega_{\mu+\psi k}\left(y_{1}, \ldots, y_{r}\right) \eta^{k} \\
& =\left(1-\frac{x t}{\beta}\right)^{1-\alpha} \exp (t) \Lambda_{\mu, \psi}\left(y_{1}, \ldots, y_{r} ; \eta\right),
\end{aligned}
$$

which completes the proof.

Now put

$$
\Omega_{\mu+\psi k}\left(y_{1}, \ldots, y_{r}\right):=\Phi_{\mu+\psi k}^{(\alpha)}\left(y_{1}, \ldots, y_{r}\right)
$$

in Theorem 2.1, where the multivariable polynomials $\Phi_{\mu+\psi k}^{(\alpha)}\left(x_{1}, \ldots, x_{r}\right)$ are generated by (see $[\mathrm{NE}]$ )

$$
\left(1-x_{1} t\right)^{-\alpha} e^{\left(x_{2}+\cdots+x_{r}\right) t}=\sum_{n=0}^{\infty} \Phi_{n}^{(\alpha)}\left(x_{1}, \ldots, x_{r}\right) t^{n}\left(\alpha \in \mathbb{C} ;|t|<\left|x_{1}\right|^{-1}\right) .
$$

Thus, we have the following result providing a class of bilateral generating functions for the multivariable polynomials $\Phi_{\mu+\psi k}^{(\alpha)}\left(x_{1}, \ldots, x_{r}\right)$ and generalized Bessel polynomials.

COROllary 2.2. If

$$
\Lambda_{\mu, \psi}\left(y_{1}, \ldots, y_{r} ; w\right):=\sum_{k=0}^{\infty} a_{k} \Phi_{\mu+\psi k}^{(\alpha)}\left(y_{1}, \ldots, y_{r}\right) w^{k} \quad\left(a_{k} \neq 0 ; \mu, \psi \in \mathbb{C}\right)
$$

then

$$
\begin{aligned}
\sum_{n=0}^{\infty} \sum_{k=0}^{[n / p]} a_{s} y_{n-p k}(x, \alpha- & n, \beta) \Phi_{\mu+\psi k}^{(\alpha)}\left(y_{1}, \ldots, y_{r}\right) \frac{w^{k}}{t^{p k}} \frac{t^{n}}{(n-p k) !} \\
= & \left(1-\frac{x t}{\beta}\right)^{1-\alpha} \exp (t) \Lambda_{\mu, \psi}\left(y_{1}, \ldots, y_{r} ; w\right)
\end{aligned}
$$

provided that the series on both sides are convergent.

REMARK 2.3. Using the generating relation (2.2) for the polynomials $\Phi_{n}^{(\alpha)}\left(x_{1}, \ldots, x_{r}\right)$ and setting $a_{k}=1, \mu=0, \psi=1$ in Corollary 2.2 , we find 
that

$$
\begin{aligned}
& \sum_{n=0}^{\infty} \sum_{k=0}^{[n / p]} y_{n-p k}(x, \alpha-n, \beta) \Phi_{k}^{(\alpha)}\left(x_{1}, \ldots, x_{r}\right) w^{k} \frac{t^{n-p k}}{(n-p k) !} \\
& =\left(1-\frac{x t}{\beta}\right)^{1-\alpha} \exp (t)\left(1-x_{1} w\right)^{-\alpha} e^{\left(x_{2}+\cdots+x_{r}\right) w}\left(\lambda \in \mathbb{C} ; \alpha_{j} \in \mathbb{C} ;|w|<\left|x_{1}\right|^{-1}\right) .
\end{aligned}
$$

THEOREM 2.4. For non-identically-vanishing functions $\Omega_{\mu}\left(y_{1}, \ldots, y_{r}\right)$ of $r$ complex variables $y_{1}, \ldots, y_{r}(r \in \mathbb{N})$, indexed by complex numbers $\mu$, let

$$
\begin{aligned}
\Lambda_{\mu, p, q}(x, \alpha-m, \beta & \left.; y_{1}, \ldots, y_{r} ; t\right) \\
& :=\sum_{k=0}^{\infty} a_{k} y_{m+q k}(x, \alpha-m, \beta) \Omega_{\mu+p k}\left(y_{1}, \ldots, y_{r}\right) \frac{t^{k}}{(k q) !},
\end{aligned}
$$

where $a_{k} \neq 0$ and

$$
\theta_{n, p, q}\left(y_{1}, \ldots, y_{r} ; \eta\right):=\sum_{k=0}^{[n / q]}\left(\begin{array}{c}
n \\
n-q k
\end{array}\right) a_{k} \Omega_{\mu+p k}\left(y_{1}, \ldots, y_{r}\right) \eta^{k} .
$$

Then for $p \in \mathbb{N}$,

$$
\begin{aligned}
& \quad \sum_{n=0}^{\infty} y_{n+m}(x, \alpha-n-m, \beta) \theta_{n, p, q}\left(y_{1}, \ldots, y_{r} ; \eta\right) \frac{t^{n}}{n !} \\
& =\left(1-\frac{x t}{\beta}\right)^{1-\alpha} \exp (t) \Lambda_{\mu, p, q}\left(x\left(1-\frac{x t}{\beta}\right)^{-1}, \alpha-m-q k, \beta ; y_{1}, \ldots, y_{r} ; \eta t^{q}\right)
\end{aligned}
$$

provided that the series on both sides are convergent.

Proof. For convenience, let $T$ denote the left-hand side of (2.4). Then

$$
T=\sum_{n=0}^{\infty} y_{n+m}(x, \alpha-n-m, \beta) \sum_{k=0}^{[n / q]}\left(\begin{array}{c}
n \\
n-q k
\end{array}\right) a_{k} \Omega_{\mu+p k}\left(y_{1}, \ldots, y_{r}\right) \eta^{k} \frac{t^{n}}{n !} .
$$

Replacing $n$ by $n+q k$ and then using 1.3 , we may write

$$
\begin{aligned}
& T=\sum_{n=0}^{\infty} \sum_{k=0}^{\infty}\left(\begin{array}{c}
n+q k \\
n
\end{array}\right) y_{n+m+q k}(, \alpha-n-q k-m, \beta) \\
& \times a_{k} \Omega_{\mu+p k}\left(y_{1}, \ldots, y_{r}\right) \eta^{k} \frac{t^{n+q k}}{(n+q k) !} \\
&=\sum_{k=0}^{\infty} a_{k}\left(\sum_{n=0}^{\infty} y_{n+m+q k}(x, \alpha-n-q k-m, \beta) \frac{t^{n}}{n !}\right) \\
& \times \Omega_{\mu+p k}\left(y_{1}, \ldots, y_{r}\right) \frac{\left(\eta t^{q}\right)^{k}}{(q k) !}
\end{aligned}
$$




$$
\begin{array}{r}
=\sum_{k=0}^{\infty} a_{k}\left[\left(1-\frac{x t}{\beta}\right)^{1-\alpha} \exp (t) y_{m+q k}\left(x\left(1-\frac{x t}{\beta}\right)^{-1}, \alpha-m-q k, \beta\right)\right] \\
\times \Omega_{\mu+p k}\left(y_{1}, \ldots, y_{r}\right) \frac{\left(\eta t^{q}\right)^{k}}{(q k) !} \\
=\left(1-\frac{x t}{\beta}\right)^{1-\alpha} \exp (t) \sum_{k=0}^{\infty} a_{k} y_{m+q k}\left(x\left(1-\frac{x t}{\beta}\right)^{-1}, \alpha-m-q k, \beta\right) \\
\times \Omega_{\mu+p k}\left(y_{1}, \ldots, y_{r}\right) \frac{\left(\eta t^{q}\right)^{k}}{(q k) !} \\
=\left(1-\frac{x t}{\beta}\right)^{1-\alpha} \exp (t) \Lambda_{\mu, p, q}\left(x\left(1-\frac{x t}{\beta}\right)^{-1}, \alpha-m-q k, \beta ; y_{1}, \ldots, y_{r} ; \eta t^{q}\right),
\end{array}
$$

which completes the proof.

If we set $s=r=1$ and $\Omega_{\mu+p k}\left(y_{1}\right)=y_{\mu+p k}\left(y, \alpha_{1}, \beta_{1}\right)$ in Theorem 2.4 where the generalized Bessel polynomials $y_{n}(x, \alpha, \beta)$ are generated by (see $[\mathrm{SM}]$ )

$$
\left(1-\frac{x t}{\beta}\right)^{1-\alpha} \exp (t)=\sum_{n=0}^{\infty} y_{n}(x, \alpha-n, \beta) \frac{t^{n}}{n !},
$$

then we get the following result.

Corollary 2.5. If

$$
\begin{array}{r}
\Lambda_{\mu, p, q}\left(x, \alpha-m, \beta ; y, \alpha_{1}, \beta_{1} ; t\right):=\sum_{k=0}^{\infty} a_{k} y_{m+q k}(x, \alpha-m, \beta) y_{\mu+p k}\left(y, \alpha_{1}, \beta_{1}\right) \frac{t^{k}}{(q k) !} \\
\left(m \in \mathbb{N}_{0}\right),
\end{array}
$$

where $a_{k} \neq 0$ for $k \neq 0$, and

$$
\theta_{n, p, q}\left(y, \alpha_{1}, \beta_{1} ; \eta\right):=\sum_{k=0}^{[n / q]}\left(\begin{array}{c}
n \\
n-q k
\end{array}\right) a_{k} y_{\mu+p k}\left(y, \alpha_{1}, \beta_{1}\right) \eta^{k}
$$

where $n, p \in \mathbb{N}$, then

$$
\begin{aligned}
& \sum_{n=0}^{\infty} y_{m+n}(x, \alpha-n-m, \beta) \theta_{n, p, q}\left(y, \alpha_{1}, \beta_{1} ; \eta\right) \frac{t^{n}}{n !} \\
= & \left(1-\frac{x t}{\beta}\right)^{1-\alpha} \exp (t) \Lambda_{\mu, p, q}\left(x\left(1-\frac{x t}{\beta}\right)^{-1}, \alpha-m-q k, \beta ; y, \alpha_{1}, \beta_{1} ; \eta t^{q}\right)
\end{aligned}
$$

provided that the series on both sides are convergent.

Furthermore, for every suitable choice of the coefficients $a_{k}\left(k \in \mathbb{N}_{0}\right)$, if the functions $\Omega_{\mu+\psi k}\left(y_{1}, \ldots, y_{r}\right), r \in \mathbb{N}$, are expressed as appropriate products of simpler functions, the assertions of Theorems 2.1 and 2.4 can be 
applied to derive various families of multilinear and multilateral generating functions for the family of generalized Bessel polynomials given explicitly by 1.1 .

3. Generalized Lauricella functions. In this section, we derive various families of bilateral generating functions for generalized Bessel polynomials and generalized Lauricella functions.

THEOREM 3.1. The following bilateral generating function holds true:

$$
\begin{aligned}
& \sum_{n=0}^{\infty} y_{n}(x, \alpha-n, \beta) \Psi_{n}\left(u_{1} ; u_{2}, \ldots, u_{s}\right) \frac{t^{n}}{n !} \\
& =\left(1-\frac{x t}{\beta}\right)^{1-\alpha} \exp (t) \sum_{m_{1}, p, m_{2}, \ldots, m_{s}=0}^{\infty} \frac{((b))_{\left(m_{1}+p\right) \phi}(\alpha-1)_{m_{1}}}{((d))_{\left(m_{1}+p\right) \delta}} \\
& \quad \times \Omega\left(f\left(m_{1}+p, m_{2}, \ldots, m_{s}\right), m_{2}, \ldots, m_{s}\right) \frac{\left(-u_{1} t\right)^{m_{1}}}{m_{1} !} \frac{\left(\frac{u_{1} x t}{x t-\beta}\right)^{p}}{p !} \frac{u_{2}^{m_{2}}}{m_{2} !} \ldots \frac{u_{s}^{m_{s}}}{m_{s} !},
\end{aligned}
$$

where $\Psi_{n}\left(u_{1} ; u_{2}, \ldots, u_{s}\right)$ is given by (1.4).

Proof. By using (1.3), we observe that

$$
\begin{aligned}
\sum_{n=0}^{\infty} y_{n}(x, \alpha-n, \beta) & \Psi_{n}\left(u_{1} ; u_{2}, \ldots, u_{s}\right) \frac{t^{n}}{n !} \\
= & \sum_{n=0}^{\infty} y_{n}(x, \alpha-n, \beta) \sum_{m_{1}=0}^{n} \sum_{m_{2}, \ldots, m_{s}=0}^{\infty} \frac{(-n)_{m_{1}}((b))_{m_{1} \phi}}{((d))_{m_{1} \delta}} \\
& \times \Omega\left(f\left(m_{1}, \ldots, m_{s}\right), m_{2}, \ldots, m_{s}\right) \frac{u_{1}^{m_{1}}}{m_{1} !} \ldots \frac{u_{s}^{m_{s}}}{m_{s} !} \frac{t^{n}}{n !} \\
= & \sum_{m_{1}, \ldots, m_{s}=0}^{\infty}\left(\sum_{n=0}^{\infty} y_{n+m_{1}}\left(x, \alpha-n-m_{1}, \beta\right) \frac{t^{n}}{n !}\right) \frac{((b))_{m_{1} \phi}}{((d))_{m_{1} \delta}} \\
& \times \Omega\left(f\left(m_{1}, \ldots, m_{s}\right), m_{2}, \ldots, m_{s}\right) \frac{\left(-u_{1} t\right)^{m_{1}}}{m_{1} !} \frac{u_{2}^{m_{2}}}{m_{2} !} \ldots \frac{u_{s}^{m_{s}}}{m_{s} !} \\
= & \sum_{m_{1}, \ldots, m_{s}=0}^{\infty} \frac{((b))_{m_{1} \phi}}{((d))_{m_{1} \delta}} \Omega\left(f\left(m_{1}, \ldots, m_{s}\right), m_{2}, \ldots, m_{s}\right) \\
& \times\left(1-\frac{x t}{\beta}\right)^{1-\alpha} \exp (t) y_{m_{1}}\left(x\left(1-\frac{x t}{\beta}\right)^{-1}, \alpha-m_{1}, \beta\right) \\
& \times \frac{\left(-u_{1} t\right)^{m_{1}}}{m_{1} !} \frac{u_{2}^{m_{2}}}{m_{2} !} \ldots \frac{u_{s}^{m_{s}}}{m_{s} !}
\end{aligned}
$$




$$
\begin{aligned}
= & \left(1-\frac{x t}{\beta}\right)^{1-\alpha} \exp (t) \sum_{m_{1}, \ldots, m_{s}=0}^{\infty} y_{m_{1}}\left(x\left(1-\frac{x t}{\beta}\right)^{-1}, \alpha-m_{1}, \beta\right) \\
& \times \frac{((b))_{m_{1} \phi}}{((d))_{m_{1} \delta}} \Omega\left(f\left(m_{1}, \ldots, m_{s}\right), m_{2}, \ldots, m_{s}\right) \frac{\left(-u_{1} t\right)^{m_{1}}}{m_{1} !} \frac{u_{2}^{m_{2}}}{m_{2} !} \ldots \frac{u_{s}^{m_{s}}}{m_{s} !} \\
= & \left(1-\frac{x t}{\beta}\right)^{1-\alpha} \exp (t) \sum_{m_{1}, \ldots, m_{s}=0}^{\infty}{ }_{2} F_{0}\left[-m_{1}, \alpha-1 ;-;-\frac{x\left(1-\frac{x t}{\beta}\right)^{-1}}{\beta}\right] \\
& \times \frac{((b))_{m_{1} \phi} \Omega\left(f\left(m_{1}, \ldots, m_{s}\right), m_{2}, \ldots, m_{s}\right) \frac{\left(-u_{1} t\right)^{m_{1}}}{m_{1} !} \frac{u_{2}^{m_{2}}}{m_{2} !} \ldots \frac{u_{s}^{m_{s}}}{m_{s} !}}{=} \\
& \left(1-\frac{x t}{\beta}\right)^{1-\alpha} \exp (t) \sum_{m_{1}, \ldots, m_{s}=0}^{\infty} \frac{((b))_{m_{1} \phi}}{((d))_{m_{1} \delta}} \Omega\left(f\left(m_{1}, \ldots, m_{s}\right), m_{2}, \ldots, m_{s}\right) \\
& \times \sum_{p=0}^{m_{1}} \frac{\left(-m_{1}\right)_{p}(\alpha-1)_{p}}{p !}\left(-\frac{x\left(1-\frac{x t}{\beta}\right)^{-1}}{\beta} \frac{\left(-u_{1} t\right)^{m_{1}}}{m_{1} !} \frac{u_{2}^{m_{2}}}{m_{2} !} \ldots \frac{u_{s}^{m_{s}}}{m_{s} !}\right. \\
= & \left(1-\frac{x t}{\beta}\right)^{1-\alpha} \exp (t) \quad \sum_{m_{1}, p, m_{2}, \ldots, m_{s}=0}^{\infty} \frac{((b))_{\left(m_{1}+p\right) \phi}(\alpha-1)_{p}}{((d))_{\left(m_{1}+p\right) \delta}} \\
& \times \Omega\left(f\left(m_{1}+p, m_{2}, \ldots, m_{s}\right), m_{2}, \ldots, m_{s}\right) \frac{\left(-u_{1} t\right)^{m_{1}}}{m_{1} !} \frac{\left(\frac{u_{1} x t}{x t-\beta}\right)^{p}}{p !} \frac{u_{2}^{m_{2}}}{m_{2} !} \ldots \frac{u_{s}^{m_{s}}}{m_{s} !},
\end{aligned}
$$

which gives the result.

By appropriately choosing $\Omega$ in Theorem 3.1, we can obtain several interesting results which give bilateral generating functions for generalized Bessel polynomials and generalized Lauricella functions.

I. Taking

$$
\begin{aligned}
\Omega\left(f\left(m_{1}, \ldots, m_{s}\right), m_{2}, \ldots, m_{s}\right) & \\
= & \frac{\prod_{j=1}^{A}\left(a_{j}\right)_{m_{1} \theta_{j}^{(1)}+\cdots+m_{s} \theta_{j}^{(s)}}}{\prod_{j=1}^{E}\left(c_{j}\right)_{m_{1} \psi_{j}^{(1)}+\cdots+m_{s} \psi_{j}^{(s)}}} \prod_{j=1}^{D^{(2)}\left(d_{j}^{(2)}\right)_{m_{2} \delta_{j}^{(2)}}} \cdots \frac{\prod_{j=1}^{B^{(2)}\left(b_{j}^{(s)}\right)_{m_{s} \phi_{j}^{(s)}}}}{\prod_{j=1}^{D^{(s)}\left(d_{j}^{(s)}\right)_{m_{s} \delta_{j}^{(s)}}}}
\end{aligned}
$$

in Theorem 3.1, we obtain the next result.

COROLlary 3.2. The following bilateral generating function holds true:

$$
\begin{aligned}
\sum_{n=0}^{\infty} y_{n}(x, \alpha-n, \beta) F_{E: D ; D^{(2)} ; \ldots ; D^{(s)}}^{A: B+1 ; B^{(2)} ; \ldots ; B^{(s)}}\left(\begin{array}{l}
{\left[(a): \theta^{(1)}, \ldots, \theta^{(s)}\right]:[-n: 1],[(b): \phi] ;} \\
{\left[(c): \psi^{(1)}, \ldots, \psi^{(s)}\right]: \quad}
\end{array}\right][(d): \delta] ; \\
{\left[\left(b^{(2)}\right): \phi^{(2)}\right] ; \ldots ;\left[\left(b^{(s)}\right): \phi^{(s)}\right] ; } \\
{\left[\left(d^{(2)}\right): \delta^{(2)}\right] ; \ldots ;\left[\left(d^{(s)}\right): \delta^{(s)}\right] ; }
\end{aligned}
$$




$$
\begin{gathered}
=\left(1-\frac{x t}{\beta}\right)^{1-\alpha} \exp (t) F_{E+D: 0 ; 0 ; D^{(2)} ; \ldots ; D^{(s)}}^{A+B: 0 ; 1 ; B^{(2)} ; \ldots ; B^{(s)}}\left(\begin{array}{c}
{\left[(e): \varphi^{(1)}, \ldots, \varphi^{(s+1)}\right]:-,[\alpha-1: 1]} \\
{\left[(f): \xi^{(1)}, \ldots, \xi^{(s+1)}\right]:-,-;} \\
{\left[\left(b^{(2)}\right): \phi^{(2)}\right] ; \ldots ;\left[\left(b^{(s)}\right): \phi^{(s)}\right] ;} \\
{\left[\left(d^{(2)}\right): \delta^{(2)}\right] ; \ldots ;\left[\left(d^{(s)}\right): \delta^{(s)}\right] ;}
\end{array}-u_{1} t, \frac{u_{1} x t}{x t-\beta}, u_{2}, \ldots, u_{s}\right),
\end{gathered}
$$

where the coefficients $e_{j}, f_{j}, \varphi_{j}^{(s)}$ and $\xi_{j}^{(s)}$ are given by

$$
\begin{gathered}
e_{j}= \begin{cases}a_{j} & (1 \leq j \leq A), \\
b_{j-A} & (A<j \leq A+B),\end{cases} \\
f_{j}= \begin{cases}c_{j} & (1 \leq j \leq E), \\
d_{j-E} & (E<j \leq E+D),\end{cases} \\
\varphi_{j}^{(r)}= \begin{cases}\theta_{j}^{(1)} & (1 \leq j \leq A ; 1 \leq r \leq 2), \\
\theta_{j}^{(r-1)} & (1 \leq j \leq A ; 2<r \leq s+1), \\
\phi_{j-A} & (A<j \leq A+B ; 1 \leq r \leq 2), \\
0 & (A<j \leq A+B ; 2<r \leq s+1),\end{cases} \\
\xi_{j}^{(r)}= \begin{cases}\psi_{j}^{(1)} & (1 \leq j \leq E ; 1 \leq r \leq 2), \\
\psi_{j}^{(r-1)} & (1 \leq j \leq E ; 2<r \leq s+1), \\
\delta_{j-E} & (E<j \leq E+D ; 1 \leq r \leq 2), \\
0 & (E<j \leq E+D ; 2<r \leq s+1) .\end{cases}
\end{gathered}
$$

II. Upon setting

$$
\Omega\left(f\left(m_{1}, \ldots, m_{s}\right), m_{2}, \ldots, m_{s}\right)=\frac{(a)_{m_{1}+\cdots+m_{s}}\left(b_{2}\right)_{m_{2}} \ldots\left(b_{s}\right)_{m_{s}}}{\left(c_{1}\right)_{m_{1}} \ldots\left(c_{s}\right)_{m_{s}}}
$$

and

$$
\phi=\delta=0 \quad\left(\text { that is, } \phi_{1}=\cdots=\phi_{B}=\delta_{1}=\cdots=\delta_{D}=0\right)
$$

in Theorem 3.1, we obtain the following result.

COROLlARY 3.3. The following bilateral generating function holds true:

$$
\begin{aligned}
\sum_{n=0}^{\infty} y_{n}(x, \alpha-n, \beta) F_{A}^{(s)}\left[a,-n, b_{2}, \ldots, b_{s} ; c_{1}, \ldots, c_{s} ; u_{1}, \ldots, u_{s}\right] \frac{t^{n}}{n !} \\
=\left(1-\frac{x t}{\beta}\right)^{1-\alpha} \exp (t) F_{1: 0 ; 0 ; 1 ; \ldots ; 1}^{1: 0 ; 1 ; 1 ; \ldots ; 1}\left(\begin{array}{c}
{[(a): 1, \ldots, 1]:-;[\alpha-1: 1] ;} \\
{\left[\left(c_{1}\right): \psi^{(1)}, \ldots, \psi^{(s+1)}\right]:-;-;} \\
{\left[b_{2}: 1\right] ; \ldots ;\left[b_{s}: 1\right] ;-u_{1} t, \frac{u_{1} x t}{x t-\beta}, u_{2}, \ldots, u_{s}} \\
{\left[c_{2}: 1\right] ; \ldots ;\left[c_{s}: 1\right] ;}
\end{array}\right),
\end{aligned}
$$


where $F_{A}^{(s)}$ is a Lauricella function of $s$ and the coefficients $\psi^{(\eta)}$ are given by

$$
\psi^{(\eta)}= \begin{cases}1 & (1 \leq \eta \leq 2), \\ 0 & (2<\eta \leq s+1) .\end{cases}
$$

Remark 3.4. In Corollary 3.3, taking $s=2$, we obtain bilateral generating function for generalized Bessel polynomials and Appell functions.

III. If we put

$\Omega\left(f\left(m_{1}, \ldots, m_{s}\right), m_{2}, \ldots, m_{s}\right)=\frac{\left(a_{1}^{(1)}\right)_{m_{2}} \ldots\left(a_{1}^{(s-1)}\right)_{m_{s}}\left(a_{2}^{(1)}\right)_{m_{2}} \ldots\left(a_{2}^{(s-1)}\right)_{m_{s}}}{(c)_{m_{1}+\cdots+m_{s}}}$ and $B=1, b_{1}=b, \phi_{1}=1$ and $\delta=0$ in Theorem 3.1. we obtain the next result.

COROLlary 3.5. The following bilateral generating function holds true:

$$
\begin{aligned}
& \sum_{n=0}^{\infty} y_{n}(x, \alpha-n, \beta) F_{B}^{(s)}\left[-n, a_{1}^{(1)}, \ldots, a_{1}^{(s-1)}, b, a_{2}^{(1)}, \ldots, a_{2}^{(s-1)} ; c ; u_{1}, \ldots, u_{s}\right] \frac{t^{n}}{n !} \\
& =\left(1-\frac{x t}{\beta}\right)^{1-\alpha} \exp (t) F_{1: 0 ; 0 ; 0 ; \ldots ; 0}^{1: 0 ; 1 ; 2 ; ; 2}\left(\begin{array}{c}
{\left[(b): \theta^{(1)}, \ldots, \theta^{(s+1)}\right]:-;[\alpha-1: 1] ;} \\
{[(c): 1, \ldots, 1]: \quad-; \quad-;}
\end{array}\right. \\
& {\left[a^{(1)}: 1\right] ; \ldots ;\left[a^{(s-1)}: 1\right] \text {; }} \\
& \left.-u_{1} t, \frac{u_{1} x t}{x t-\beta}, u_{2}, \ldots, u_{s}\right),
\end{aligned}
$$

where $F_{B}^{(s)}$ is a Lauricella function of $s$ and the coefficients $\theta^{(\eta)}$ are given by

$$
\theta^{(\eta)}= \begin{cases}1 & (1 \leq \eta \leq 2), \\ 0 & (2<\eta \leq s+1) .\end{cases}
$$

Remark 3.6. In Corollary 3.5, putting $s=2$, we obtain bilateral generating function for generalized Bessel polynomials and Appell functions.

IV. By letting

$$
\Omega\left(f\left(m_{1}, \ldots, m_{s}\right), m_{2}, \ldots, m_{s}\right)=\frac{(a)_{m_{1}+\cdots+m_{s}}\left(b_{2}\right)_{m_{2}} \ldots\left(b_{s}\right)_{m_{s}}}{(c)_{m_{1}+\cdots+m_{s}}}
$$

and $\phi=\delta=0$ in Theorem 3.1, we obtain the following result.

COROLlary 3.7. The following bilateral generating function holds true:

$$
\begin{aligned}
& \sum_{n=0}^{\infty} y_{n}(x, \alpha-n, \beta) F_{D}^{(s)}\left[a,-n, b_{2}, \ldots, b_{s} ; c ; u_{1}, u_{2}, \ldots, u_{s}\right] \frac{t^{n}}{n !} \\
& =\left(1-\frac{x t}{\beta}\right)^{1-\alpha} \exp (t) F_{D}^{(s+1)}\left[a, \alpha-1, b_{2}, \ldots, b_{s} ; c ;-u_{1} t, \frac{u_{1} x t}{x t-\beta}, u_{2}, \ldots, u_{s}\right],
\end{aligned}
$$

where $F_{D}^{(s)}$ is a Lauricella function of $s$. 
REMARK 3.8. In Corollary 3.7, taking $s=2$, we get a bilateral generating function for generalized Bessel polynomials and Appell functions.

4. Miscellaneous properties. In this section we give some properties of the generalized Bessel polynomials $y_{n}(x, \alpha, \beta)$.

TheOREM 4.1. The generalized Bessel polynomials $y_{n}(x, \alpha, \beta)$ have the following integral representation:

$$
y_{n}(x, \alpha-n, \beta)=\frac{1}{\Gamma(\alpha-1)} \int_{0}^{\infty} e^{-u} u^{\alpha-2}\left(1+\frac{x}{\beta} u\right)^{n} d u
$$

when $\operatorname{Re}(\alpha-1)>0$.

Proof. If we use the identity

$$
a^{-v}=\frac{1}{\Gamma(v)} \int_{0}^{\infty} e^{-a t} t^{v-1} d t \quad(\operatorname{Re}(v)>0)
$$

on the left-hand side of 1.2 , we have

$$
\begin{aligned}
\sum_{n=0}^{\infty} y_{n}(x, \alpha-n, \beta) \frac{t^{n}}{n !} & =\left(1-\frac{x t}{\beta}\right)^{1-\alpha} \exp (t) \\
& =\left(\frac{1}{\Gamma(\alpha-1)} \int_{0}^{\infty} e^{-\left(1-\frac{x t}{\beta}\right) u} u^{\alpha-1-1} d u\right) \exp (t) \\
& =\frac{1}{\Gamma(\alpha-1)} \int_{0}^{\infty} e^{-u} u^{\alpha-2} e^{t+\frac{x t}{\beta} u} d u \\
& =\frac{1}{\Gamma(\alpha-1)} \int_{0}^{\infty} e^{-u} u^{\alpha-2} e^{\left(1+\frac{x}{\beta} u\right) t} d u \\
& =\frac{1}{\Gamma(\alpha-1)} \int_{0}^{\infty} e^{-u} u^{\alpha-2} \sum_{n=0}^{\infty} \frac{\left(1+\frac{x}{\beta} u\right)^{n}}{n !} t^{n} d u
\end{aligned}
$$

From the coefficients of $t^{n}$ on both sides, one gets the desired result.

We now discuss some miscellaneous recurrence relations for generalized Bessel polynomials. By differentiating both sides of 1.2 with respect to $x$ and using

$$
\sum_{n=0}^{\infty} \sum_{k=0}^{\infty} A(k, n)=\sum_{n=0}^{\infty} \sum_{k=0}^{n} A(k, n-k),
$$

we arrive at the following (differential) recurrence relation for generalized 
Bessel polynomials:

$y_{n}^{\prime}(x, \alpha-n, \beta)=\sum_{m=0}^{n-1} \frac{(-n)_{m}(1-\alpha)(-x)^{m}}{\beta^{m+1}} y_{n-m-1}(x, \alpha-n+m+1, \beta), n \geq 1$.

Moreover, by differentiating each side of 1.2 with respect to $t$, we have another recurrence relation for these polynomials:

$$
\begin{aligned}
y_{n+1}(x, \alpha-n-1, \beta) & =y_{n}(x, \alpha-n, \beta) \\
+ & \sum_{m=0}^{n}(-1)^{m}(-n)_{m} \frac{(\alpha-1) x^{m+1}}{\beta^{m+1}} y_{n-m}(x, \alpha-n+m, \beta) .
\end{aligned}
$$

Acknowledgements. The author would like to thank the reviewers for their careful reading of the manuscript.

\section{References}

[RAB] R. Aktaş, A. Altın and B. Cekim, On a two-variable analogue of Bessel functions, J. Inequal. Spec. Funct. 3 (2012), 13-23.

[AE] A. Altın and E. Erkus,, On a multivariable extension of the Lagrange-Hermite polynomials, Integral Transforms Spec. Funct. 17 (2006), 239-244.

[BSF] M. G. de Bruin, E. B. Saff and R. S. Varga, On the zeros of generalized Bessel polynomials I, Nederl. Akad. Wetensch. Indag. Math. 43 (1981), 1-13.

[CV] A. J. Carpenter, R. S. Varga and J. Waldvogel, Asymptotics for the zeros of the partial sums of $e^{z}$, Rocky Mountain J. Math. 21 (1991), 99-120.

[BE] B. Cekim and E. Erkuş-Duman, Integral representations for Bessel matrix functions, Gazi Univ. J. Sci. 27 (2014), 663-667.

[CS] M.-P. Chen and H. M. Srivastava, A note on certain generating functions for the generalized Bessel polynomials, J. Math. Anal. Appl. 180 (1993), 151-159.

[DSS] G. Dattoli, H. M. Srivastava and D. Sacchetti, A note on the Bessel polynomials, Math. Maced. 1 (2003), 1-7.

[EAR] E. Erkuş-Duman, A. Altın and R. Aktaş, Miscellaneous properties of some multivariable polynomials, Math. Computer Modelling 54 (2011), 1875-1885.

[CM] C. Kaanoğlu and M. A. Özarslan, Two-parameter Srivastava polynomials and several series identities, Adv. Difference Equations 2013, art. 81, 9 pp.

[H] H. L. Krall and O. Frink, A new class of orthogonal polynomials: the Bessel polynomials, Trans. Amer. Math. Soc. 65 (1949), 100-115.

[L] G. Lauricella, Sulle funzioni ipergeometriche a più variabili, Rend. Circ. Mat. Palermo 7 (1893), 111-158.

[SL] S.-J. Liu, Bilateral generating functions for the Lagrange polynomials and the Lauricella functions, Integral Transforms Spec. Funct. 20 (2009), 519-527.

[CCS] S.-J. Liu, C.-J. Chyan, H.-C. Lu, and H. M. Srivastava, Bilateral generating functions for the Chan-Chyan-Srivastava polynomials and the generalized Lauricella functions, Integral Transforms Spec. Funct. 23 (2012), 539-549.

[SSHM] S.-J. Liu, S.-D. Lin, H. M. Srivastava and M.-M. Wong, Bilateral generating functions for the Erkus-Srivastava polynomials and the generalized Lauricella functions, Appl. Math. Comput. 218 (2012), 7685-7693. 
[N] N. Özmen, Some new properties of the Meixner polynomials, Sakarya Univ. J. Sci. 21 (2017), 1454-1462.

[NE] N. Özmen and E. Erkuş-Duman, Some results for a family of multivariable polynomials, AIP Conf. Proc. 1558 (2013), 1124-1127.

[NE*] N. Özmen and E. Erkuş-Duman, On the Poisson-Charlier polynomials, Serdica Math. J. 41 (2015), 457-470.

[OE] N. Özmen and E. Erkuş-Duman, Some families of generating functions for the generalized Cesàro polynomials, J. Comput. Anal. Appl. 25 (2018), 670-683.

[SM] H. M. Srivastava and H. L. Manocha, A Treatise on Generating Functions, Horwood and Halsted Press, 1984.

[SD] H. M. Srivastava and M. C. Daoust, Certain generalized Neumann expansions associated with the Kampé de Fériet function, Nederl. Akad. Wetensch. Proc. Ser. A 72 = Indag. Math. 31 (1969), 449-457.

Nejla Özmen

Department of Mathematics

Faculty of Science and Arts

Düzce University

Düzce, Turkey

E-mail: nejlaozmen06@gmail.com nejlaozmen@duzce.edu.tr 